\title{
The oncolytic peptide LTX-315 overcomes resistance of cancers to immunotherapy with CTLA4 checkpoint blockade
}

\author{
T Yamazaki ${ }^{1,2,3}$, JM Pitt ${ }^{1,2,3}$, M Vétizou $^{1,2,3}$, A Marabelle $^{1,2}$, C Flores $^{1,2,3}, \emptyset$ Rekdal $^{4,5}$, G Kroemer $^{\star, 3,6,7,7,8,9,10,11,12,13}$ and \\ L Zitvogel $\left.\right|^{\star, 1,2,3,9,12,13}$
}

Intratumoral immunotherapies aim at reducing local immunosuppression, as well as reinstating and enhancing systemic anticancer T-cell functions, without inducing side effects. LTX-315 is a first-in-class oncolytic peptide-based local immunotherapy that meets these criteria by inducing a type of malignant cell death that elicits anticancer immune responses. Here, we show that LTX-315 rapidly reprograms the tumor microenvironment by decreasing the local abundance of immunosuppressive Tregs and myeloid-derived suppressor cells and by increasing the frequency of polyfunctional T helper type 1/type 1 cytotoxic T cells with a concomitant increase in cytotoxic T-lymphocyte antigen-4 (CTLA4) and drop in PD-1 expression levels. Logically, in tumors that were resistant to intratumoral or systemic CTLA4 blockade, subsequent local inoculation of LTX-315 cured the animals or caused tumor regressions with abscopal effects. This synergistic interaction between CTLA4 blockade and LTX-315 was reduced upon blockade of the $\beta$-chain of the interleukin-2 receptor (CD122). This preclinical study provides a strong rationale for administering the oncolytic peptide LTX-315 to patients who are receiving treatment with the CTLA4 blocking antibody ipilimumab.

Cell Death and Differentiation (2016) 23, 1004-1015; doi:10.1038/cdd.2016.35; published online 15 April 2016

Local immunotherapy of cancer capable of mediating abscopal effects - and hence long-term protection from metastatic relapse - represents a holy grail in oncology. ${ }^{1}$ Indeed, in as much as favorable immune contextures of primary tumors were found to be associated with long-term protection of the cancer bearer, ${ }^{2,3}$ one can assume that stimulating local immunity in primary or metastatic tumors may reinstate systemic anticancer immune responses eliminating against all deposits of neoplastic cells. Marabelle et al. ${ }^{4}$ revealed that a combination of various immunomodulators could be injected into tumors resulting in the eradication of local regulatory $\mathrm{T}$ cells (Tregs) ${ }^{5,6}$ culminating in the control of disseminated tumors. Similar results were obtained by combining local radiotherapy with systemic anti-cytotoxic T-lymphocyte antigen-4 (anti-CTLA4) antibodies, ${ }^{7}$ which may mediate the FcyR-dependent elimination of Tregs in murine and perhaps human cancers. ${ }^{8}$ Few local immunotherapies have reached the market with the notable exception of talimogene laherparepvec (T-VEC), a transgenic oncolytic virus expressing granulocyte-macrophage colony-stimulating factor, which received Food and Drug Administration (FDA) approval for surgically unresectable skin and lymph node lesions in patients with advanced melanoma. However, no effect on melanoma that have spread to internal organs and no increase in survival were reported for T-VEC ${ }^{9}$ calling for the development of improved therapies.

Cationic antimicrobial peptides are small molecules found in a large diversity of species including bacteria, fungi, plants and animals. ${ }^{10}$ Although extensively varying in their amino-acid sequences and structural motifs, they have recurrent structural and functional properties such as cationicity and amphipathicity enabling disruption of lipid membranes. Some cationic antimicrobial peptides exhibit lipopolysaccharideneutralizing activities ${ }^{11,12}$ and immune modulatory effects. ${ }^{13}$ In addition to their well-recognized antimicrobial killing capacities, several cationic antimicrobial peptides also

${ }^{1}$ Institut National de la Santé et de la Recherche Médicale (INSERM), U1015, Equipe Labellisée Ligue Nationale Contre le Cancer, Gustave Roussy Cancer Campus, Villejuif, France; ${ }^{2}$ Université Paris Descartes, Sorbonne Paris Cité, Paris, France; ${ }^{3}$ Université Paris Sud, Paris Saclay, Le Kremlin-Bicêtre, France; ${ }^{4}$ University of Tromsø, Tromsø, Norway; ${ }^{5}$ Lytix Biopharma AS, Tromsø, Norway; ${ }^{6}$ Equipe 11 Labellisée par la Ligue Contre le Cancer, Centre de Recherche des Cordeliers, Paris, France; ${ }^{7}$ Cell Biology and Metabolomics platforms, Gustave Roussy Comprehensive Cancer Center, Villejuif, France; ${ }^{8}$ INSERM, U1138, Paris, France; ${ }^{9}$ Université Pierre et Marie Curie, Paris, France; ${ }^{10}$ Pôle de Biologie, Hôpital Européen Georges Pompidou, AP-HP, Paris, France; ${ }^{11}$ Department of Women's and Children's Health, Karolinska Institute, Karolinska University Hospital, Stockholm, Sweden and ${ }^{12}$ Center of Clinical Investigations in Biotherapies of Cancer (CICBT1428), Villejuif, France

*Corresponding author: G Kroemer or L Zitvogel, INSERM U1015, Equipe Labellisée Ligue Nationale Contre le Cancer, Gustave Roussy Cancer Campus, 114, rue Edouard Vaillant, Villejuif 94800, France. E-mail: Kroemer@orange.fr or Laurence.zitvogel@gustaveroussy.fr

${ }^{13}$ Shared co-last authorship.

Abbreviations: TLR, toll-like receptor; CTLA4, cytotoxic T-lymphocyte antigen-4; FDA, food and drug administration; Tc1, type 1 cytotoxic T cell; Th1, Thelper type 1; Tr1, type 1 regulatory T-cell; Treg, regulatory T cell; WT, wild type; HMGB1, high-mobility group box 1; CXCL10, (C-X-C motif) ligand 10; MDSC, myeloid-derived suppressor cell; TILs, intratumoral lymphocytes; MTD, minimal tumorigenic dose; CTL, cytotoxic T lymphocyte; IFNAR1, interferon- $\alpha / \beta$ receptor; MCA, methylcholanthrene; ZFN, zincfinger nuclease; mAb, monoclonal antibody; ICB, immune checkpoint blocker; ICD, immunogenic cell death; DC, dendritic cell; APC, antigen-presenting cell; Lag3, lymphocyte-activation gene 3; sCD25, soluble CD25

Received 07.12.15; revised 29.2.16; accepted 08.3.16; Edited by S Fulda; published online 15.4.16 
displayed tumoricidal activities. ${ }^{14-17}$ Bovine lactoferricin is a cationic antimicrobial peptide isolated from cow's milk after acid-pepsin hydrolysis of the lactoferrin protein ${ }^{18}$ endowed with potent cytotoxicity in vitro and against a variety of murine and human cancer cell lines, and in vivo against subcutaneous or metastatic tumor deposits. ${ }^{19-24}$ The chemical modifications of bovine lactoferricin derivatives allowed for the development of a lead compound, LTX-315 with a shorter chemically modified peptide length and optimal (selective) anticancer properties. ${ }^{25-29}$

By creating pores and disintegrating the cytoplasmic membranes, as well as targeting the mitochondria, LTX-315 promotes the release of damage-associated molecular patterns (DAMPs) associated with immunogenic cell death (ICD). ${ }^{26,27,29}$ Transmission electron microscopy or morphometric analysis of chromatin-stained tumor cells revealed that LTX-315 failed to induce classical 'apoptosis' associated with apoptotic nuclear condensation and caspase-3-dependent cell death. Rather, the oncolytic peptide-mediated cell death exhibited a necrotic phenotype that was not regulated in as much as neither necrostatin-1 nor cyclosporin A compromised cell death. ${ }^{30}$

Phase I clinical trials were conducted in advanced stage cancer patients, using intralesional and serial administrations of LTX-315. Complete and partial regressions were observed in $33 \%(6 / 18)$ of injected lesions with concomitant infiltration of cytotoxic CD8 ${ }^{+}$T cells in 50\% (5/10) patients undergoing serial biopsies. Disease stabilization was recorded in $75 \%(6 / 8)$ of the patients. ${ }^{31}$ We already reported that LTX-315-mediated tumor cell death exhibited immunostimulatory properties in various tumor models. ${ }^{26,27,29}$ Here, we show that, in contrast to prototypic cytotoxicants such as anthracyclines, LTX-315associated cell death does not require type 1 interferon $1 a / \beta$ receptor (IFNAR), Toll-like receptor-3 (TLR3) and TLR4 signaling to mediate its long-term protective antitumor effects. However, LTX-315 drastically reduced Treg and myeloidderived suppressor cell (MDSC) locally, inducing the accumulation of polyfunctional interferon- $\gamma(\mathrm{IFN} \gamma)^{+} \mathrm{TNF}^{+} \mathrm{T}$ cells in tumor beds (intratumoral lymphocytes (TILs)), culminating in the improvement of the ratio of $\mathrm{CD}^{+} \mathrm{T}$ effector cell ratio over Tregs. Importantly, these polyfunctional $\mathrm{CD}^{+}$TILs did not acquire the exhaustion marker programmed death-1 (PD-1) during this activation step, and rather increased the expression of cytotoxic T-lymphocyte-associated protein 4 on their surface (CTLA4). In tumors resistant to intratumoral or systemic CTLA4 blockade, subsequent local inoculations of LTX-315 caused profound tumor regressions and even cures, accompanied by abscopal effects (meaning that even malignant lesions that were established in the opposite flank and were not treated by LTX-315 responded to the therapy). In similar settings, LTX-315 failed to synergize with antiprogrammed cell death-1 antibodies in inhibiting the growth of similar cancers. Interestingly, the antitumor effects of LTX-315, as well as its synergy with anti-CTLA4 antibody $(A b)$, both depended upon the interleukin-2 receptor $\beta$ chain (CDD122), which is required for signaling in response to interleukin-2 (IL-2) and IL-15. These preclinical findings highlight new prospects for the future development of local immunotherapies.

\section{Results}

LTX-315 induced T-cell-dependent antitumor and abscopal effects. Day 7 established MCA205 sarcoma reaching 20-25 mm $\mathrm{mm}^{2}$ sizes in syngeneic C57BL/6 mice were treated with three daily intratumoral administrations of LTX-315. All tumor-bearing mice were cured (Figure 1a, left and middle panel) and immunized against syngeneic sarcoma (Figure 1a, right panel), in as much as a rechallenge with five times the minimal tumorigenic dose (MTD) of MCA205, which was lethal in control mice (data not shown), failed to grow in LTX-315-treated littermates. In contrast, the latter animals succumbed to a rechallenge with irrelevant EL4 syngeneic lymphoma cells (Figure 1a, right panel). On larger sarcoma $\left(>40 \mathrm{~mm}^{2}\right)$, LTX-315 could also control tumor progression (growth kinetics, Figure 1b, left panel and tumor weight, Figure 1b, right panel) and exhibited abscopal effects by impacting distant tumor deposits (Figure 1c), suggesting a protective role of adaptive immune responses. LTX-315-mediated anticancer effects were T-cell dependent in as much as antibodies depleting $\mathrm{CD}^{+}$and $\mathrm{CD}^{+} \mathrm{T}$ cells completely abrogated the antitumor effects (Figure 1d). We confirmed the efficacy of the oncolytic peptide in less immunogenic transplantable tumor models, such as B16F10 subcutaneous melanoma (Figure 1e). Altogether, LTX-315 locally acted as an immunogenic cytotoxic compound in vivo.

LTX-315 increased the intratumoral ratio of cytotoxic T lymphocytes (CTLs) over Tregs. We next explored the dynamics of the main immunosuppressors and effectors composing the tumor microenvironment shaped 7 days post LTX-315 in the subcutaneous sarcoma model. A modest increase in $\mathrm{CD} 45^{+}$leukocytic cells was observed but there was a significant increase in $\mathrm{CD}^{+}$leukocytes (Figure 2a) including $\mathrm{CD}^{+} \mathrm{T}$ and $\mathrm{CD}^{+} \mathrm{T}$ lymphocytes (Figure $2 \mathrm{~b}$ ). Importantly, myeloid-derived Ly6C ${ }^{\text {low }} \mathrm{CD} 11 \mathrm{~b}^{+}$cells and regulatory $\mathrm{CD}^{+} \mathrm{T}$ cells, defined as $\mathrm{CD}^{+} 5^{+} \mathrm{FoxP}^{+}$or $\mathrm{OX} 40^{+}$ $\mathrm{CTLA}^{+}$expressing TILs, were markedly decreased by LTX-315 (Figure 2c and data not shown). The activation profile of intratumoral dendritic cells (DCs) defined as CD11 ${ }^{+}$ $\mathrm{I}-\mathrm{A} / \mathrm{I}-\mathrm{E}^{+} \mathrm{CD} 11 \mathrm{~b}^{+}$cells did not significantly change with this local therapy (Supplementary Figure 1). In parallel, we observed an accumulation of $\mathrm{IFN} \gamma^{+}$( $\mathrm{T}$ helper type 1, Th1), $\mathrm{IL}-17^{+}$(Th17) and double-positive IFN $\gamma^{+} \mathrm{IL}-17^{+}$(pTh17) $\mathrm{CD}^{+}$ TILs (Figure 2d), as well as polyfunctional IFN $\gamma^{+} \mathrm{TNFa}^{+} \mathrm{CD}^{+}$ $\mathrm{T}$ cells (Figure $2 \mathrm{e}$ ). Not surprisingly, the ratio of $\mathrm{CD}^{+} \mathrm{IFN} \gamma^{+}$ $\mathrm{T}$ cells over Tregs, as well as the ratio of $\mathrm{CD}^{+} \mathrm{TNFa}^{+} \mathrm{T}$ cells over Tregs, markedly increased post LTX-315 (Figure 2f). Altogether, we conclude that the oncolytic peptide is capable of rapidly reprogramming the tumor microenvironment of an established tumor, decreasing immunosuppressive cells while increasing polyfunctional Th1/type 1 cytotoxic T cells.

IFNAR1, TLR3 and TLR4 are dispensable for LTX-315mediated antitumor effects. We previously reported that CTL responses against tumor antigens can be elicited after ICD induced by anthracyclines or oxaliplatin. In the context of ICD, nuclear high-mobility group box 1 (HMGB1) is released 

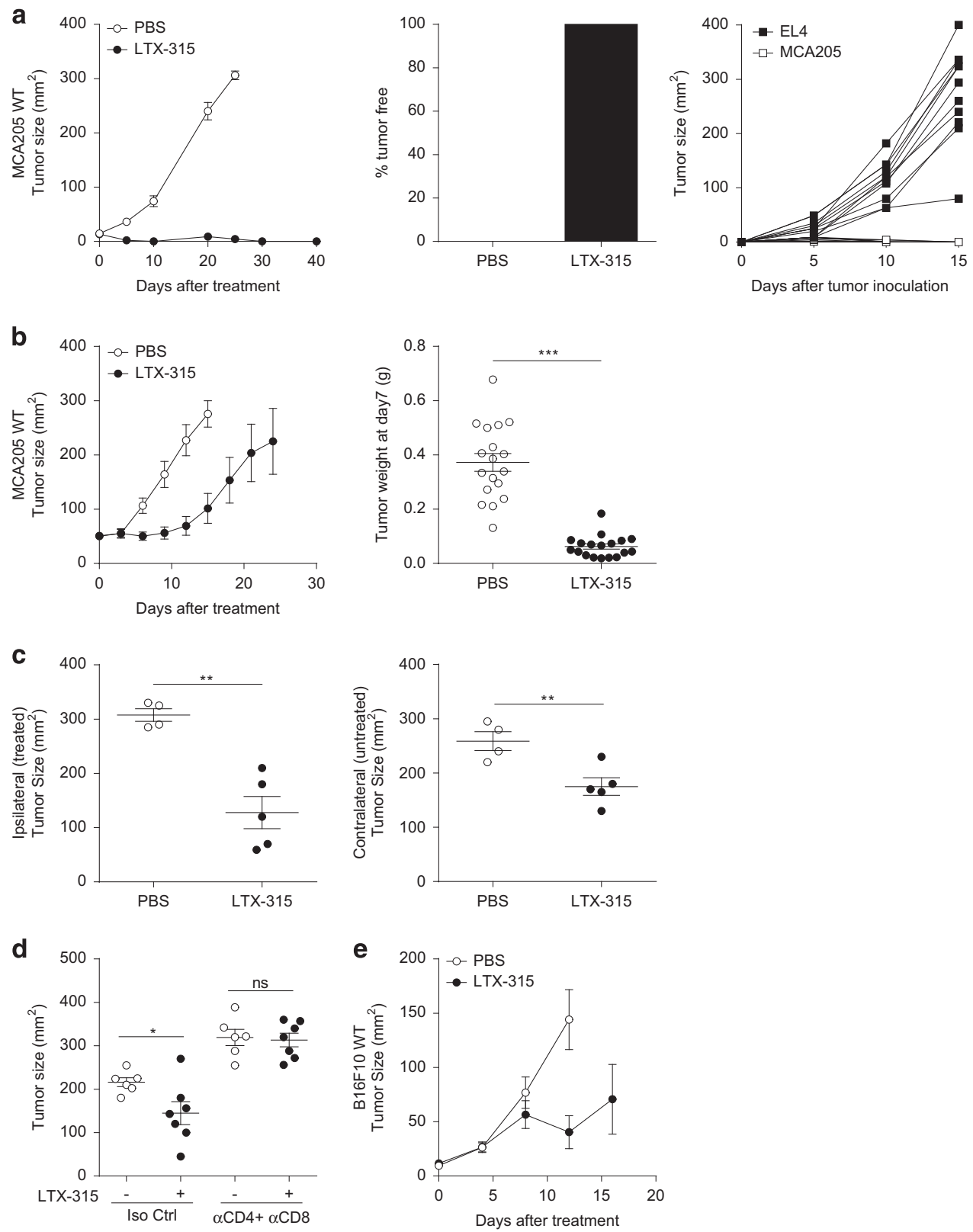

Figure 1 T-cell-dependent antitumor effects of LTX-315. (a) Anticancer activity of LTX-315 activity on small MCA205 sarcoma. Three intratumoral daily consecutive injections

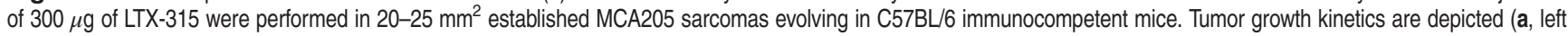
panel). The percentages of tumor-free mice is shown at day 60 (a, middle panel). After a rechallenge of tumor-free mice with the MTD of EL4 or MCA205, the tumor growth were monitored over time (a, right panel). (b) Idem as in a but treating bigger tumors of $35-40 \mathrm{~mm}^{2}$ size. Tumor growth kinetics (left panel) and weight at killing (right panel) are depicted. (c) Bilateral tumor inoculation in the right and left flank four days apart and local injection of LTX-315 only in the first (older, day 8 established) tumor. Tumor sizes for all animals are shown at killing in the ipsilateral (treated, left panel) and contralateral (untreated, right panel) sites. (d) Effects of depleting antibodies targeting CD4 and CD8a molecules on the tumoricidal activity of LTX-315. Tumor growth kinetics in the presence or absence of such specific antibodies (GK1.5 and 53-6.72, 200 $\mu \mathrm{g}$ per mouse) or isotype control mAbs injected. (e) LTX-315 antitumor effects against B16F10 s.c. melanoma. Idem as in a (left panel) following inoculation of the MTD of B16F10. In all graphs, a representative experiment out of two comprising 6-7 mice per group is shown. Student's $t$-test: ${ }^{*} P<0.05,{ }^{* *} P<0.01,{ }^{* *} P<0.001$; NS, not significant

during the necrotic phase of apoptosis to facilitate TLR4mediated antigen processing in surrounding antigenpresenting cells (APCs). ${ }^{32}$ Moreover, type 1 IFN is secreted for $24-48 \mathrm{~h}$ by cancer cells subsequent to the stimulation of TLR3 to act in a paracrine manner on cancer cell-associated IFNAR, thereby stimulating the release of the chemokine (C-X-C motif) ligand 10 (Cxcl10) and the CXCR3-mediated recruitment of T lymphocytes into tumor beds. ${ }^{33}$ Hence, we analyzed the release of these two major hallmarks of ICD, that is, the alarmin HMGB1 and the chemokine Cxcl10 in vitro $30 \mathrm{~min}$ after exposure to low dosing of the cationic antimicrobial peptide. A very low dose $(25 \mu \mathrm{g} / \mathrm{ml})$ of LTX-315 was sufficient to trigger the release/secretion of both HMGB1 and Cxcl10 (Figure 3a). Despite this release, 
host-derived TLR4 (the receptor for HMGB1) or interferon$a / \beta$ receptor (IFNAR1; the receptor functionally upstream of Cxcl10 production) appeared dispensable for the anticancer efficacy of LTX-315 (Figure 3b, left and right panels) on CT26 and MCA205 cancers. Finally, we analyzed the impact of cell autonomous IFNAR or TLR3 signaling pathways on the a

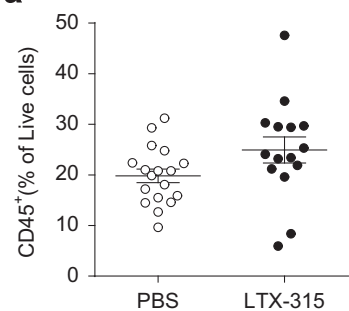

C

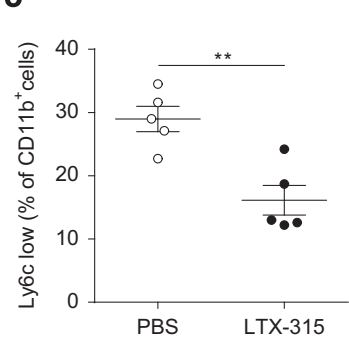

d
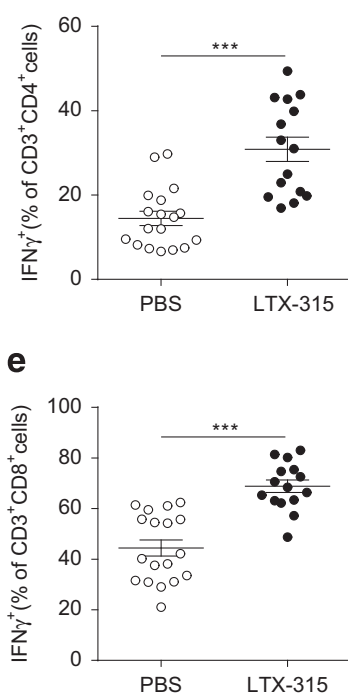

f

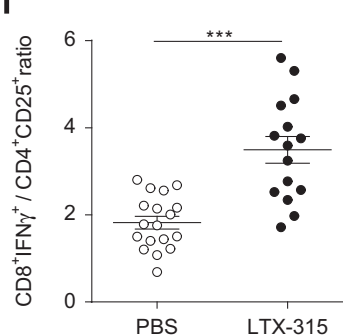

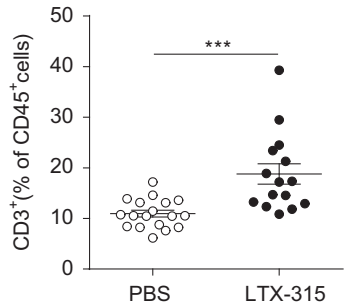
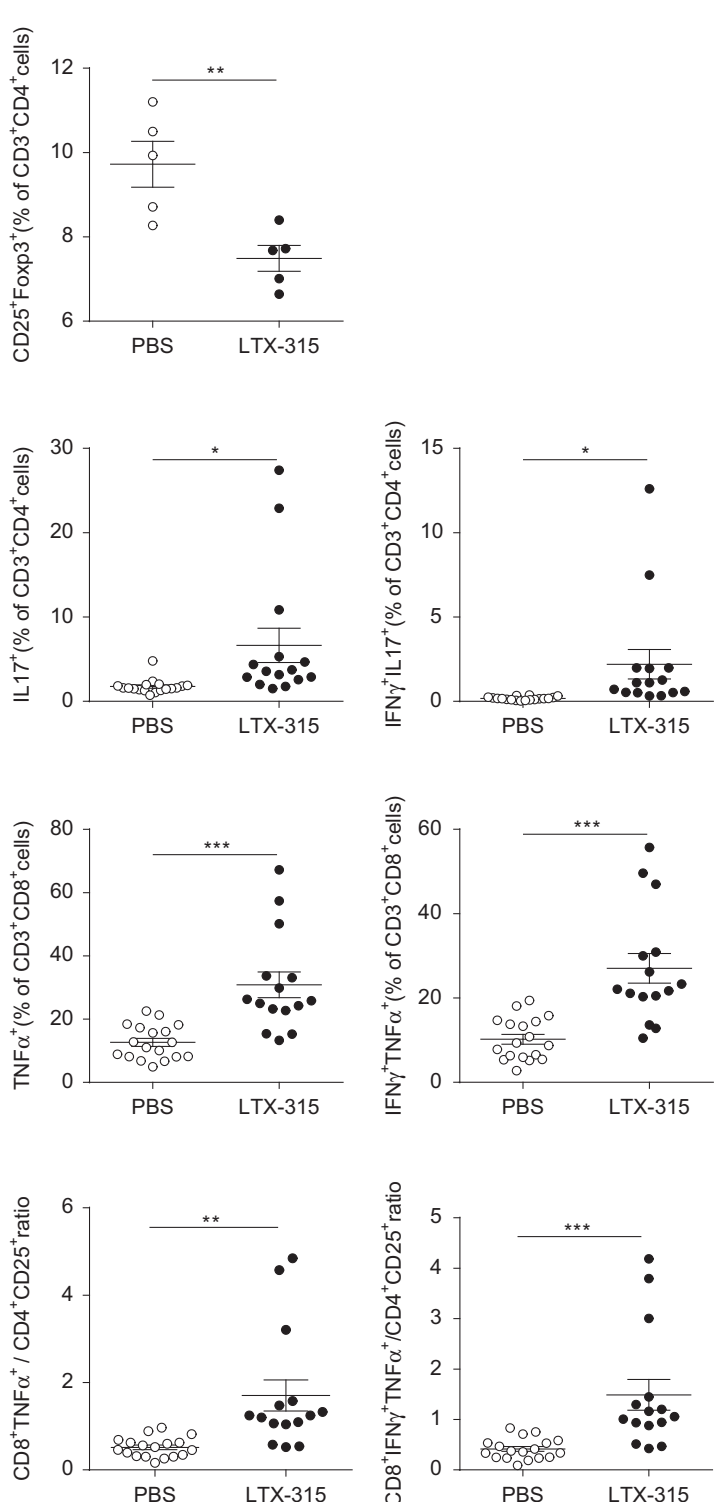

b

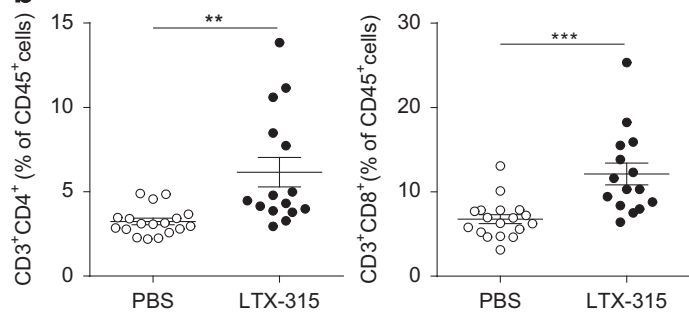

PBS LTX-315

Figure 2 LTX-315 markedly increased the CTL/Treg ratio in tumor beds. Flow cytometry determination of CD45 leukocytes (a, left panel) in the gate of live cells after dissociation of fresh MCA205 sarcoma 7 days post LTX-315 (versus PBS), as well as CD3 $3^{+}$T cells (a, right panel), CD4 ${ }^{+}$T, CD8 ${ }^{+}$T cells in the CD45 live cells (b), of CD4 $4^{+}$Treg defined as $\mathrm{CD}_{25} 5^{+} \mathrm{FoxP} 3^{+}$cells (c, right panel) and MDSC defined as CD11b ${ }^{+} \mathrm{Ly} 6 \mathrm{C}^{\text {low }}$ cells in the $\mathrm{CD} 45^{+}$gate (c, left panel), of cytokine producing $\mathrm{CD} 4^{+} \mathrm{T}$ cells in the $\mathrm{CD} 4^{+} \mathrm{T}-\mathrm{cell}$ gate (d), of cytokine producing $\mathrm{CD} 8^{+} \mathrm{T}$ cells in the $\mathrm{CD} 8^{+} \mathrm{T}$-cell gate (e). The ratio between Tc1 cells over Treg was calculated considering either IFN $\gamma^{+}$or TNF $\alpha^{+}$or double-positive $\mathrm{CD} 8^{+}$TILs (f, three panels). Each dot represents data of one mouse; at least two experiments were gathered in each graph. Student's $t$-test: ${ }^{*} P<0.05,{ }^{* \star} P<0.01,{ }^{* * *} P<0.001$; NS, not significant 
a

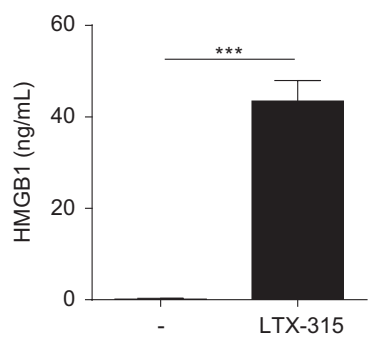

b
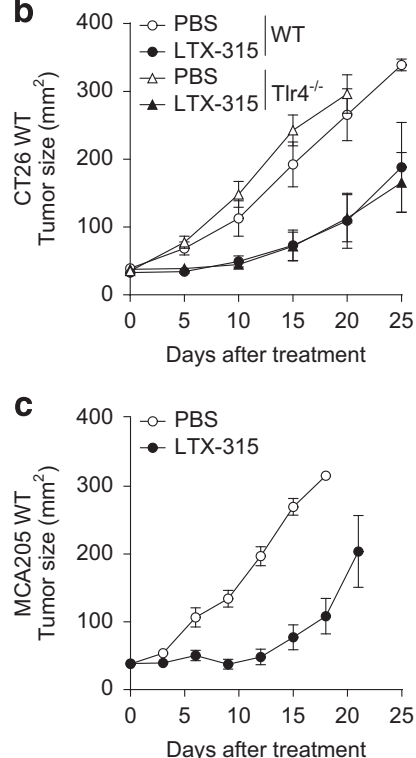
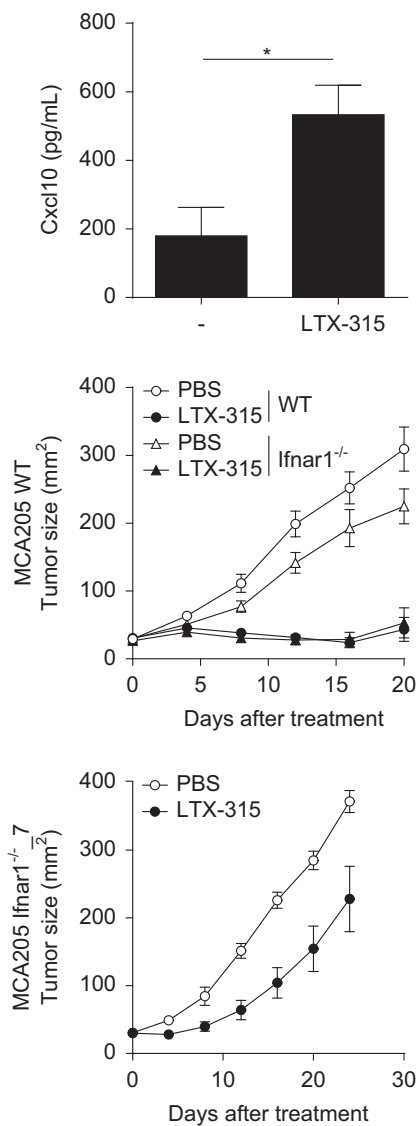

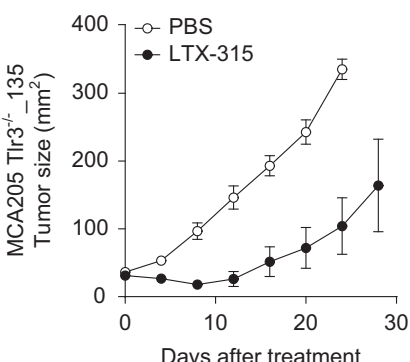

Figure 3 TLR4 and IFNAR are dispensable for the immunogenicity of LTX-315-mediated cell death. (a) HMGB1 (left) and Cxcl10 (right) release monitored by commercial ELISA 30 min after incubation with $25 \mu \mathrm{g} / \mathrm{ml}$ of LTX-315. The depicted results represent two independent experiments performed in triplicate wells. (b) LTX-315 tumoricidal activity in TLR4 (left panel) and IFNAR1 (right panel) loss-of-function mice bearing a day 8 established CT26 colon cancer or MCA205 sarcoma respectively. (c) Bioactivity of LTX-315 on several clones of established MCA205 sarcoma rendered genetically defective by means of zinc-finger nucleases (ZFN). For (b and $\mathbf{c}$ ), similar experimental settings as in Figure 1a (left panel) were applied. In all graphs, a representative experiment out of two comprising 6-7 mice per group is shown. Student's $t$-test: ${ }^{*} P<0.05$, ${ }^{* \star} P<0.001$

oncolytic activity of the peptide in established methylcholanthrene (MCA)-induced sarcoma clones. Independent MCA-induced sarcoma obtained from wild-type (WT; clone 6) or Ifnar2 $^{-1}$ (clone 4) mice responded equally well to the oncolytic peptide when implanted into WT mice (Supplementary Figures $2 a$ and $b$ ), in conditions in which anthracyclines failed to reduce the growth of clone $4 .^{33}$ Similarly, the IFNAR1-deficient MCA205 clone 7 and 19-14 (resulting from nucleotransfection with specific zinc-finger nucleases (ZFN) designed to target the Ifnar1 gene) did respond to LTX-315 while it failed to respond to doxorubicin in vivo (Figure $3 \mathrm{c}$ and Supplementary Figure 2b). ${ }^{33}$ Altogether, these results indicate that IFNAR does not need to be expressed by tumor nor by host cells to facilitate LTX-315-elicited anticancer immune responses. Similarly to TLR3-sufficient control tumors (clone 6), one MCA-induced sarcoma from $\mathrm{Tlr}^{-/}$origin (clone 4) inoculated into WT recipients as well as two independent MCA205 clone 135 and 206 resulting from nucleotransfection with specific ZFN designed to target T/r3 gene (resulting in targeted deletion of the T/r3 gene, as shown in western blot) ${ }^{33}$ were still controlled by LTX-315 in vivo (Figure 3c and Supplementary Figure $2 \mathrm{c}$ ). In brief, in contrast to anthracyclines, LTX-315 did not require the HMGB1/TLR4 and IFNAR/TLR3 transduction pathways to induce tumoricidal activity in vivo.

LTX-315 differentially modulated immune checkpoint receptors in TILs: impact on the scheduling of combinatorial regimen. Immune checkpoint blockers (ICBs) have been added to the oncological armamentarium following the FDA approval of therapeutic antibodies directed against CTLA4 and PD-1 for the treatment of selected malignant diseases. These monoclonal antibodies (mAbs) aim at priming cancer-specific T-cell responses (as for CTLA4 blockade) or reactivating exhausted TILs (for PD-1 blockade). Therefore, on theoretical grounds, systemic administration of mAb targeting these immune checkpoints could be advantageously combined with local immunotherapies such as LTX-315. We first analyzed how such immune checkpoints were regulated by local injection of the oncolytic peptide at the peak of the intratumoral T-cell response (7 days post LTX-315). Membrane CTLA4 molecules were clearly upregulated by the peptide, both with respect to the percentages of $\mathrm{CTLA}^{+}$cells and mean fluorescence intensities in $\mathrm{CD}^{+}$(data not shown) and CTL (Figure 4a). In sharp contrast, there was a statistically significant and 

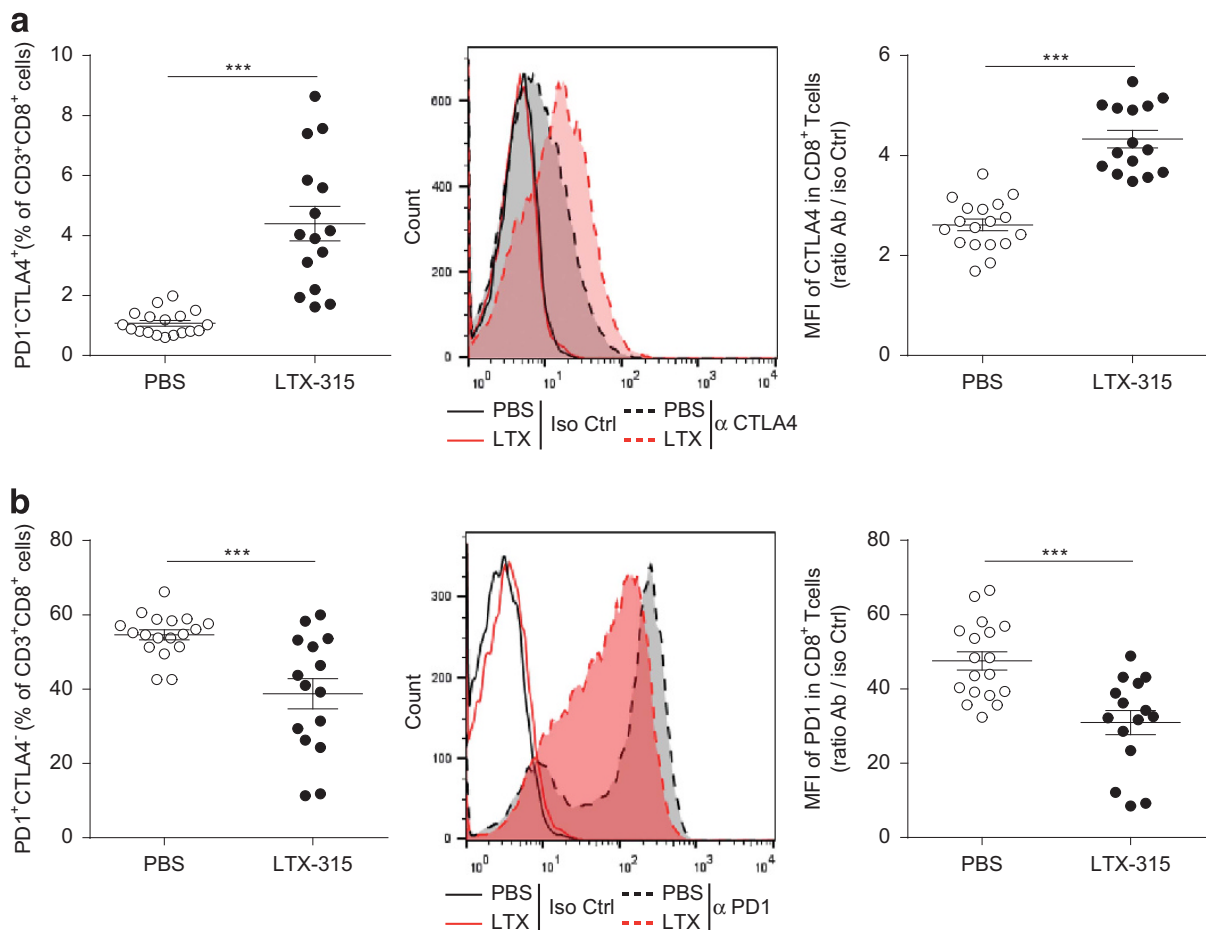

Figure 4 LTX-315 affected T-cell surface expression of the major immune checkpoints. Flow cytometry determination of CTLA4 (a) or PD-1 (b) molecules on CD8 ${ }^{+}$TILs at day 7 post LTX-315 (versus PBS) local injections. Percentages of positive cells (left panels) or mean fluorescence intensities (MFI) (middle and right panels) shown in overlay graphs or ratios of MFI between specific versus isotype control mAb are depicted. Each dot represents one mouse; two experiments were gathered in each graph. Student's $t$-test: ${ }^{* * *} P<0.001$

concomitant reduction of PD-1 expression on $\mathrm{CD}^{+}$TILs (Figure 4b)

These findings prompted us to compare the relative efficacy of anti-CTLA4 versus anti-PD-1 mAb in boosting the immunedependent antitumor effects of local LTX-315. However, scheduling, dosing and routing remain three pivotal issues when it comes to designing optimal combinatorial regimen. We first analyzed how ICB would boost LTX-315-induced anticancer activity by starting ICB, 7 days after the last LTX-315 intratumoral inoculations (Figure 5a). In a first set of experiments performed on day 7 established MCA205 sarcomas, adding PD-1 mAb failed to ameliorate LTX-315mediated tumor control. Up to $17 \%$ of the LTX-315-treated animals were tumor free at the time of killing, irrespective of the treatment with PD-1 blockade (Figure $5 b$ ). In a second set of experiments performed on smaller tumors (i.e., $20-25 \mathrm{~mm}^{2}$ ), LTX-315 alone was more efficient, leading to 33\% complete tumor rejections (calculated from all replicate experiments combined), yet the addition of CTLA4 blockade at later time points failed to significantly ameliorate tumor growth kinetics and cure rates (Figure $5 \mathrm{c}$, leading to cure in $50 \%$ of the mice). Next, we started with ICB treatments and administered the oncolytic peptide 7 days later (Figure 6a). PD-1 blockade followed by LTX-315 had minimal effects on the natural growth of sarcomas (Figure 6b). In sharp contrast, an initial treatment with CTLA4 blockade followed by LTX-315 7 days later revealed a major therapeutic effect (with cure in $50 \%$ of the cases). It is importantly to stress that day 8 sarcomas were quite resistant to monotherapy with CTLA4 blockade, whereas LTX-315 alone was no longer effective against large established sarcomas on day 15. However, the combination of both treatments exhibited a major synergistic interaction (Figure 6c).

We next applied both therapies, anti-CTLA4 mAb on day 8 followed by LTX-315 on day 15 in the form of local injections, a modality that enabled us to reduce the amount of anti-CTLA4 $m A b$ required for therapeutic effects by a factor of three (Figure 7a). Moreover, we addressed the question as to whether this new regimen could mediate abscopal effects on distant tumor deposits. For this, tumors were implanted in the right and left flanks 4 days apart, but only one of the tumors was injected locally ('ipsilateral [treated]'). We obtained a strong therapeutic synergy between CTLA4 blockade and LTX-315, both on ipsilateral and contralateral MCA205 sarcomas, when tumor sizes, cure rates or survival curves were computed (Figures $7 \mathrm{~b}-\mathrm{d}$ ). Blockade with anti-CTLA4 alone was seen to result in slightly greater control of contralateral tumor than ipsilateral tumor growth, this most likely due to the contralateral tumor being 4 days less advanced and hence likely having a less-established immunosuppressive microenvironment. Altogether, we conclude that LTX-315 was capable of markedly enhancing the immunemediated anticancer effects induced by CTLA4 blockade, even in tumors that were primarily resistant to antiCTLA4 mAb.

Role of CD122 in the synergistic effect of CTLA4 blockade and LTX-315. We previously reported that the efficacy of anti-CTLA4 mAb relied upon signaling of either IL-2 or IL-15 on the common IL-2/IL-15 receptor $\beta$ chain 
a

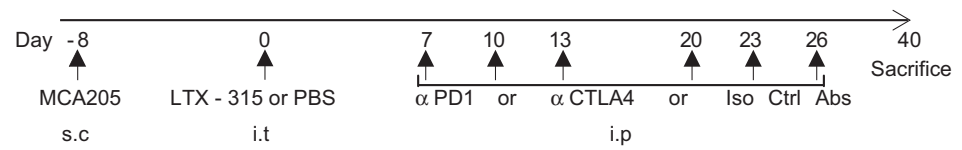

b
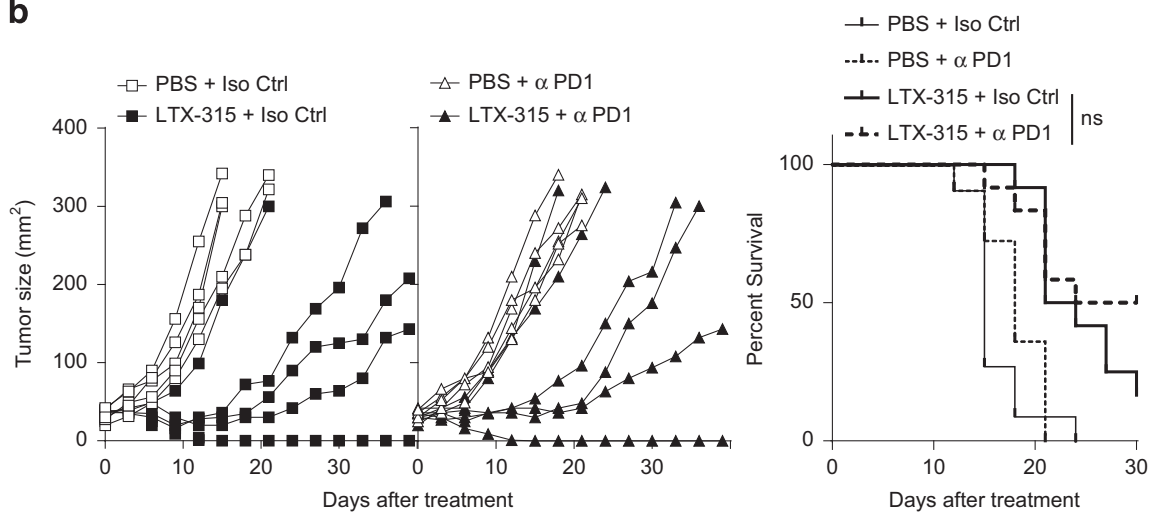

C
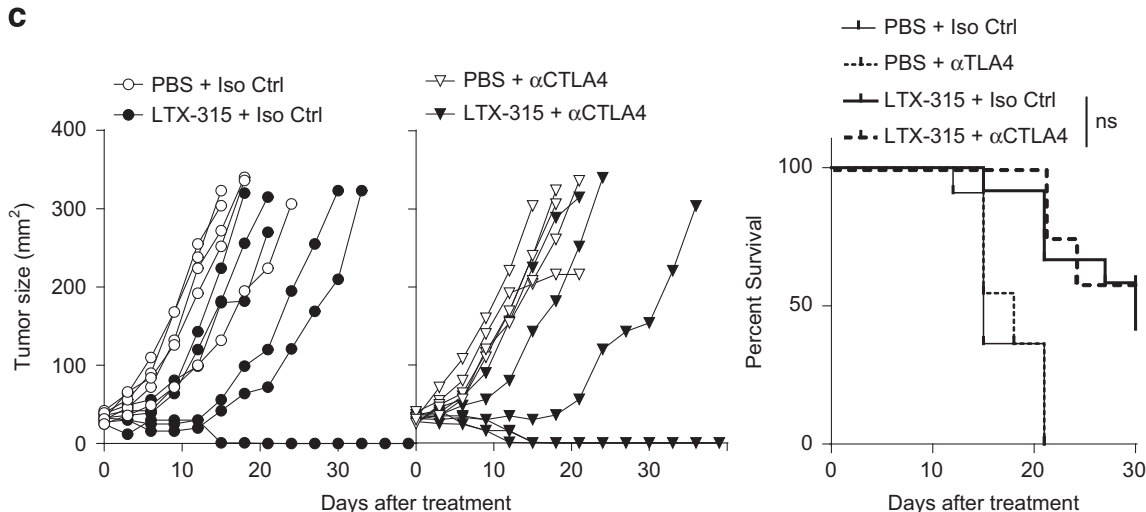

Figure 5 The LTX-315-induced tumoricidal activity is not markedly boosted by ICB. (a) Experimental setting. Scheduling and routing of the combination therapy with LTX-315 injected first locally and ICB injected sequentially in systemic administrations as detailed in Materials and Methods section. s.c., subcutaneous; i.p., intraperitoneal; i.t., intratumoral. (b and $\mathbf{c}$ ) Tumor growth kinetics in the presence (right panel) or absence (left panel) of such immune checkpoint-targeting antibodies (anti-CTLA4: $100 \mu \mathrm{g} / \mathrm{mouse}$ or anti-PD-1 mAb: $250 \mu \mathrm{g} /$ mouse, three injections weekly for 2 weeks) or isotype control mAbs injected after LTX-315. The Kaplan-Meier curves are depicted in the right panels gathering two experiments. In the graphs for ( $\mathbf{b}$ and $\mathbf{c}$ ), one representative out of two identically performed experiments comprising 6-7 mice per group is shown for the left and middle panels depicting tumor growth curves (conclusions drawn from total dataset from both replicates), and all data combined are shown in the right panels depicting Kaplan-Meier survival. Comparisons of Kaplan-Meier survival curves were performed using the log-rank Mantel-Cox test; NS, not significant

(CD122), meaning that neutralization of any of these molecules blunted the immune-dependent antitumor efficacy of anti-CTLA4 mAb. ${ }^{34}$ We therefore evaluated whether blockade of CD122 would abolish the local or abscopal therapeutic effects of LTX-315, alone or in combination with CTLA4 blockade (Figure 8a).

On the treated side, the LTX-315 mediated a CD122dependent antitumor effect (Figures $8 \mathrm{~b}, P<0.01$ ) in a context where anti-CD122 mAb did not impact the natural immunosurveillance of this tumor. Anti-CTLA4 mAb alone exerted a significant but not impressive tumor control $(P<0.05)$ that was CD122 independent. However, the combination of both reagents (LTX-315+anti-CD122 mAb) was synergistic leading to $18 \%$ cures and this local synergistic activity was partially abolished by anti-CD122 neutralizing mAb $(P<0.001)$.

On the contralateral tumor deposit, CTLA4 blockade was more effective than LTX-315 (Figure $8 \mathrm{c}, P<0.05$ ) in a CD122-dependent manner (as previously described). ${ }^{34}$ The
LTX-315-mediated abscopal effects were also significantly blunted by the anti-CD122 mAb (Figure 8c, $P<0.05$ ). The tumoricidal activity of the combination of both reagents was also partially impaired by the anti-CD122 mAb (Figure 8c, $P<0.05)$.

Altogether, neutralizing anti-CD122 mAb mostly reduced the local and abscopal anticancer effects of LTX-315, be it alone or in combination with anti-CTLA4 mAb. Hence, some of the therapeutic mechanisms of action established for CTLA4 blockade alone may also be relevant for LTX-315 and contribute to the bioactivity of the combination regimen.

\section{Discussion}

LTX-315 is a cationic antimicrobial peptide with oncolytic properties following intralesional administration in mice and humans. It mediates its anticancer activity against a wide variety of histological tumor types in a T-cell-dependent 
a

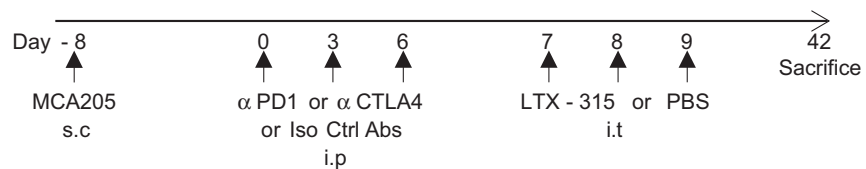

b

i.p
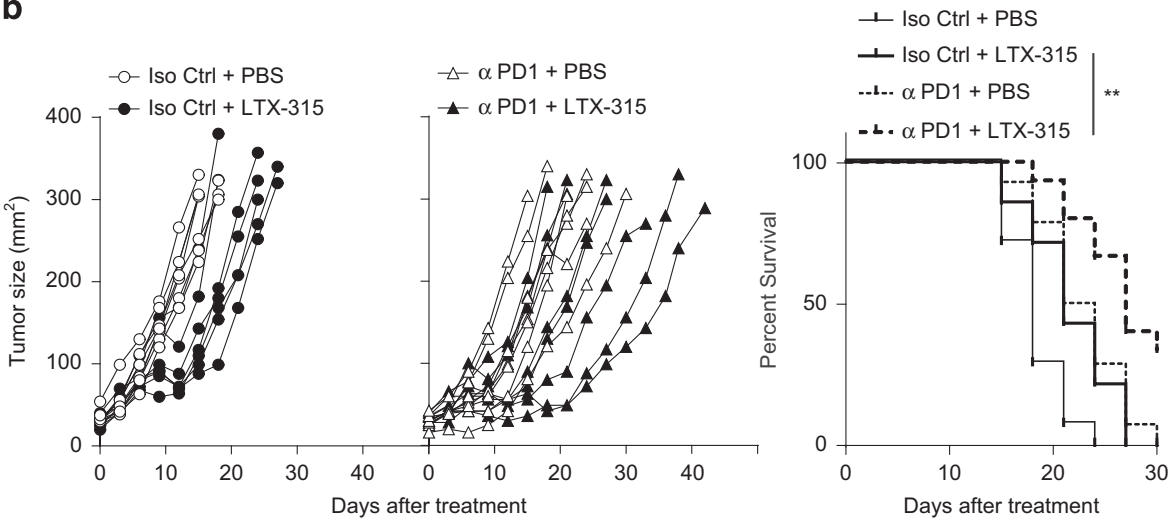

C
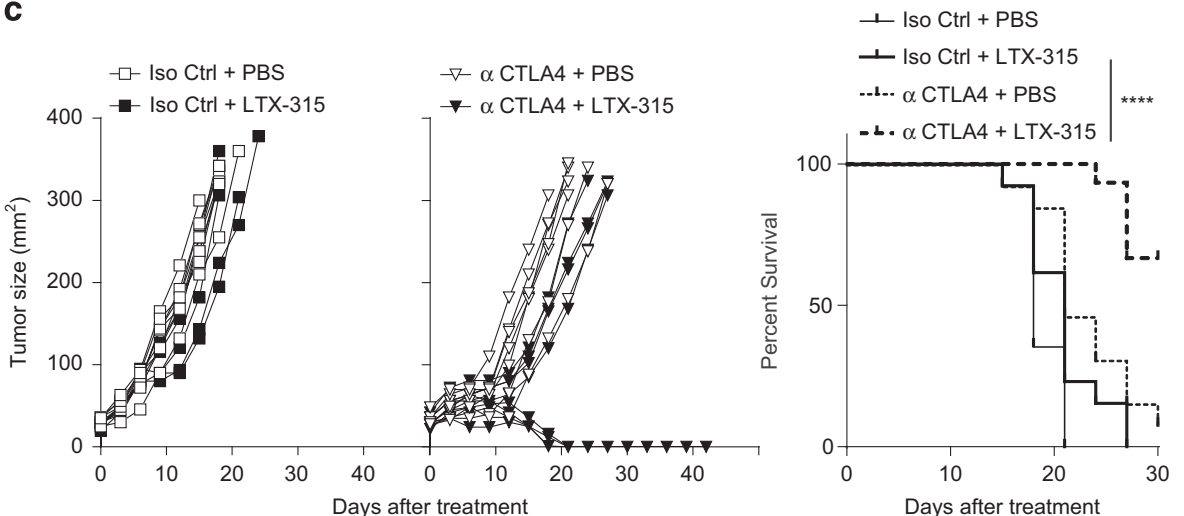

Figure 6 CTLA4 blockade markedly synergized with subsequent local delivery of LTX-315. (a) Experimental setting. Scheduling and routing of mAbs injections as detailed in Materials and Methods section. S.c., subcutaneous; i.p., intraperitoneal; i.t., intratumoral. (b and c) Tumor growth kinetics in the presence (right panel) or absence (left panel) of such immune checkpoint-targeting antibodies (anti-CTLA4: $100 \mu \mathrm{g} /$ mouse or anti-PD-1 mAb: $250 \mu \mathrm{g} /$ mouse, three injections each) or isotype control mAbs injected before LTX-315. The Kaplan-Meier curves are depicted in the right panels gathering two experiments. In all graphs, a representative experiment out of two comprising 6-7 mice per group is shown. Comparison of Kaplan-Meier survival curves were performed using the log-rank Mantel-Cox test: ${ }^{* \star} P<0.01$, ${ }^{\star \star \star \star} P<0.0001$; NS, not significant

manner by inducing cell death endowed with immunogenic properties. $^{25-35}$ In this preclinical study, we found that LTX-315 can be active against sarcomas that poorly respond to CTLA4 blockade, and that the sequential combination of anti-CTLA4 mAb followed by LTX-315 is synergistic, either using systemic or local delivery of anti-CTLA4 mAb, with a mechanism involving CD122 receptors.

LTX-315 does not induce a typical apoptotic or regulated necrotic cell death. ${ }^{30}$ However, this uncontrolled tumor cell death is 'immunogenic' in as far as (i) injection of dead cells could prevent the outgrowth of a live minimal tumorigenic dose of syngeneic tumor cells; ${ }^{28,29}$ (ii) antitumor effects were abolished in the absence of T lymphocytes; (iii) cured tumorfree mice resisted rechallenge with autologous tumor cells of the same kind; (iv) the growth kinetics of distant tumor deposits were also affected by LTX-315; (v) anti-CTLA4 antibodies set the stage for LTX-315 to be an even more potent anticancer agent; (vi) full therapeutic efficacy of LTX-315 and LTX-315 plus CTLA4 blockade depended on CD122. Although LTX-315 can trigger the hallmarks of ICD, such as the exodus of HMGB1 and the release of the Th1 chemokine Cxcl10, LTX-315 differs in its mode of action from anthracyclines or oxaliplatin $^{33}$ as LTX-315 does not require a series of cytokine and pathogen recognition receptors that are necessary for anthracyclines or oxaliplatin to stimulate anticancer immune responses. Thus, the HMGB1 host receptor TLR4 was dispensable for LTX-315-mediated tumor control. Moreover, the TLR3 and IFNAR signaling pathways were not mandatory for its bioactivity, in contrast to what we described for the long-term anticancer effects of doxorubicin or mitoxantrone. ${ }^{33}$ In contrast, we showed that LTX-315 could somehow shape the tumor microenvironment by reducing the infiltration by MDSCs and Tregs, facilitating the accumulation of polyfunctional $\mathrm{CD}^{+}$and $\mathrm{CD}^{+}$TILs. Importantly, activated TILs exhibited lower expression levels of PD-1 in this context, a phenomenon that we failed to observe with chemotherapy 
(data not shown). This may be explained by the decreased Treg proportions, which reportedly contribute to maintain PD-1 expression levels in effector T cells ${ }^{36}$ and may set the stage for a better response to PD-1 blockade in patients. Indeed, Ngiow et al. $^{36}$ highlighted the crucial role of the Treg/PD-1/PDL-1 rheostat accounting for a better antitumor activity of anti-PD-1 $\mathrm{mAb}$ in tumors containing CD8 ${ }^{+} \mathrm{PD}-1^{\text {int }} \mathrm{T}$ cells. However, in contrast to PD-1 expression, CTLA4 membrane levels substantially increased post LTX-315 treatment, which may be as a result of the strong T-cell receptor engagement following cross-presentation of dead bodies by local APCs and/or IL-2 production by polyfunctional T cells. In accordance with these observations, CTLA4 blockade appeared to be slightly more effective than PD-1 blockade after LTX-315 activity. Current studies evaluate the role of additional hallmarks of ICD such as the formyl peptide receptor and its soluble ligand annexin A1 (produced by tumor cells) ${ }^{37}$ that may explain the chemotactic activity of DC toward dying bodies for accelerated antigen processing and presentation operating during LTX-315 administration. Regardless of these mechanistic considerations, these findings prompt a concomitant administration of CTLA4 mAb with LTX-315.

On theoretical grounds, transforming tumors into their own vaccine is not only a new paradigm for effective immunomodulation but should represent an ideal strategy to mount a systemic cognate immunity that will be protective over long durations without triggering major side effects. ${ }^{1}$ Local immunotherapy should rely on four criteria to be successful. First, it should deplete Tregs, either by means of antibodydependent cell cytotoxicity, or by direct elimination, by acting through the differential threshold of toxicity in between effector and Treg cells (such as that obtained with cyclophosphamide, discussed). In that regard, LTX-315 could reduce Tregs and spare conventional polyfunctional $T$ cells. Second, local immunotherapy should promote the release of tumor antigens and DAMPs to chemoattract bona fide APCs that should process and cross-present tumor antigens. Although we did not observe a major increase in $\mathrm{CD} 11 \mathrm{C}^{+} \mathrm{I}-\mathrm{A} / \mathrm{I}-\mathrm{E}^{+} \mathrm{APC}$ in tumor beds (Supplementary Figure 1), we cannot exclude the possibility that LTX-315 could boost the capacity of APC to increase their direct or indirect participation in anticancer immunosurveillance. ${ }^{38,39}$ Third, the local immunotherapy should provide agonists for pattern recognition receptors (PRRs) to boost APC functions. Indeed, LTX-315 could provide the release of DAMPs (such as adenosine triphosphate). ${ }^{27,28}$ In this context, it may be interesting to evaluate the possibility to boost LTX-315 anticancer activity by locally administered agonists of PRR receptors that thus far have not been involved in ICD or LTX-315 effects such as TLR7-8 agonist. Fourth, engaging activating receptors or neutralizing inhibitory receptors should reinstate local or regional effector $\mathrm{T}$ and NK cells. Agonistic CD40 antibodies, or antagonistic antiglucocorticoid-induced tumor necrosis factor receptor-related protein, lymphocyte-activation gene 3 (Lag3), TIGIT or killercell immunoglobulin-like receptor are commonly tested alone or in conjunction with $\mathrm{PD}-1$ blockade to improve TIL reactivity. We observed that CTLA4 was markedly upregulated following LTX-315 activity and that blockade of this immune checkpoint set the stage of a better efficacy of LTX-315. This may be due to the conversion of $\mathrm{Lag3}^{+} \mathrm{FoxP}^{+} \mathrm{IL}-10$-producing $\mathrm{CD}^{+}$
T cells known to be suppressive through an Egr2-dependent mechanism into IL-2-secreting helper $\mathrm{CD}^{+}{ }^{+} \mathrm{TILs}^{34}$ during CTLA4 blockade. Indeed, our initial work showed that the IL-2/ IL-15/CD122 axis was mandatory for the immunostimulatory effects of anti-CTLA4 antibodies in mice and that soluble CD25 (sCD25) facilitated the accumulation of Treg cells and represented a predictive marker of resistance to ipilimumab in metastatic melanoma. ${ }^{34}$ Hence, it will be interesting to analyze whether LTX-315 bioactivity can be suppressed by sCD25 in mice and humans, and boosted by recombinant interleukin-2 or IL-15 in preclinical models.

Beyond these considerations, along with others, we have shown that a number of immunomodulators act through the intervention of the gut microbiota. ${ }^{40-43}$ In as much as LTX-315 is a cationic antimicrobial peptide, it is conceivable that it could directly affect tissue residing commensals ${ }^{44}$ or distant intestinal microflora, hereby generating some systemic adjuvanticity.

In conclusion, this work demonstrates that LTX-315 has the potential to stimulate therapeutically relevant anticancer immune responses in several preclinical models. One of the salient features of LTX-315 is that it can be administered locally, by injection into malignant lesions to locally stimulate anticancer immune responses that suppress the growth of distant tumors, and hence mediate abscopal responses. Moreover, we have accumulated data suggesting that LTX-315 can be advantageously combined with CTLA4 blockade, in particular if CTLA4 blockade precedes or is concomitant to the local administration of LTX-315.

\section{Materials and Methods}

Chemicals and cell cultures. Media and supplements for cell culture were obtained from Gibco-Life Technologies (Carlsbad, CA, USA), chemicals from Sigma-Aldrich (St. Louis, MO, USA) with the exception of LTX-315 that was provided by Lytix Biopharma (Tromsø, Norway) and plasticware from Corning BV Life Sciences (Amsterdam, The Netherlands). MCA205 and B16F10 were cultured in RPMI-1640 medium supplemented with $10 \%$ fetal calf serum, and $2 \mathrm{mM}$ L-glutamine, $100 \mathrm{IU} / \mathrm{ml}$ penicillin G sodium salt, $100 \mu \mathrm{g} / \mathrm{ml}$ streptomycin sulfate, $1 \mathrm{mM}$ sodium pyruvate and $1 \mathrm{mM}$ nonessential amino acids. Cells were grown at $37^{\circ} \mathrm{C}$ in a humidified incubator under a $5 \% \mathrm{CO}_{2}$ atmosphere.

Mice. Mice were maintained in specific pathogen-free conditions in a temperaturecontrolled environment with 12-h light, 12-h dark cycles and received food and water ad libitum. Animal experiments followed the Federation of European Laboratory Animal Science Association (FELASA) guidelines, were in compliance with EU Directive 63/2010 and were approved by the Ethical Committee of the Gustave Roussy Cancer Campus (Villejuif, France). Mice were used between 7 and 14 weeks of age. WT-specific pathogen-free (SPF) C57BL/6 J and BALB/c mice were obtained from Envigo (Gannat, France) and Janvier (Saint-Berthevin, France), respectively, and were kept in SPF conditions at Gustave Roussy, Villejuif, France. TIr $4^{-/-}$BALB/C and Ifnar1 ${ }^{-/-}$C57BL/6 mice were bred and maintained in the animal facility of Gustave Roussy. Ifnar1 ${ }^{-1-} \mathrm{C} 57 \mathrm{BL} / 6$ mice were kindly provided by the University of Montpellier (Montpellier, France) and the Peter MacCallum Cancer Centre.

Tumor models. Mice were subcutaneously injected into the right flank with $1 \times 10^{6} \mathrm{WT}$, Ifnar1 ${ }^{-/-}$or $\mathrm{TIr}^{-/-} \mathrm{MCA} 205$, or $5 \times 10^{4} \mathrm{~B} 16 \mathrm{~F} 10$ cells, or $5 \times 10^{6} \mathrm{WT}$, Ifnar2 ${ }^{-\alpha}$ or $\mathrm{Tlr}^{-/-} \mathrm{MCA}$ cells. Tumor cell lines were inoculated into WT or Ifnar1 ${ }^{-/-}$ C57BL/6 mice. In a similar model, $1 \times 10^{6}$ CT26 cells were inoculated subcutaneously (right flank) into WT or TIr4 ${ }^{-/}$BALB/c mice. Tumor surfaces (longest dimension $\times$ perpendicular dimension) were routinely monitored by caliper. When tumors reached a size of $20-40 \mathrm{~mm}^{2}$ (day 0 ), mice were administered intratumorally with three consecutive daily injections of $300 \mu \mathrm{g} \mathrm{LTX-315.} \mathrm{In} \mathrm{other}$ settings, mice were injected intraperitoneally (i.p.) with $100 \mu \mathrm{g}$ of anti-CTLA4 mAb 
a

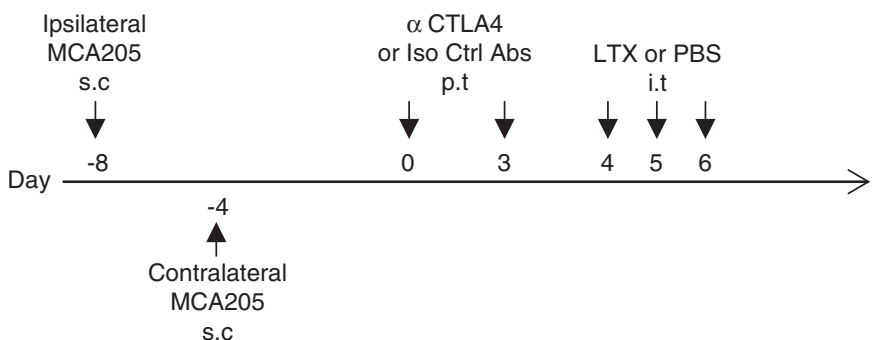

b
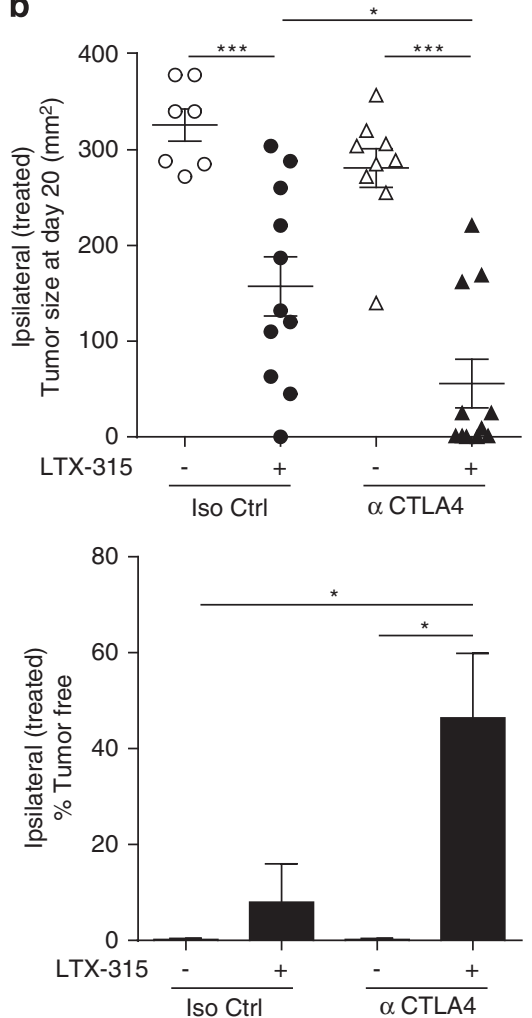

d
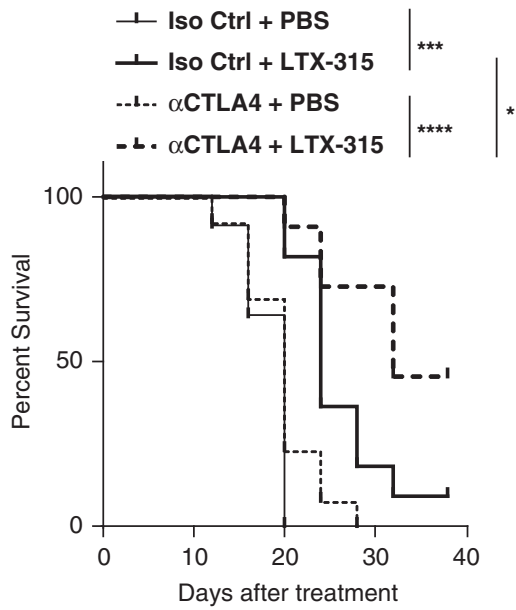

C
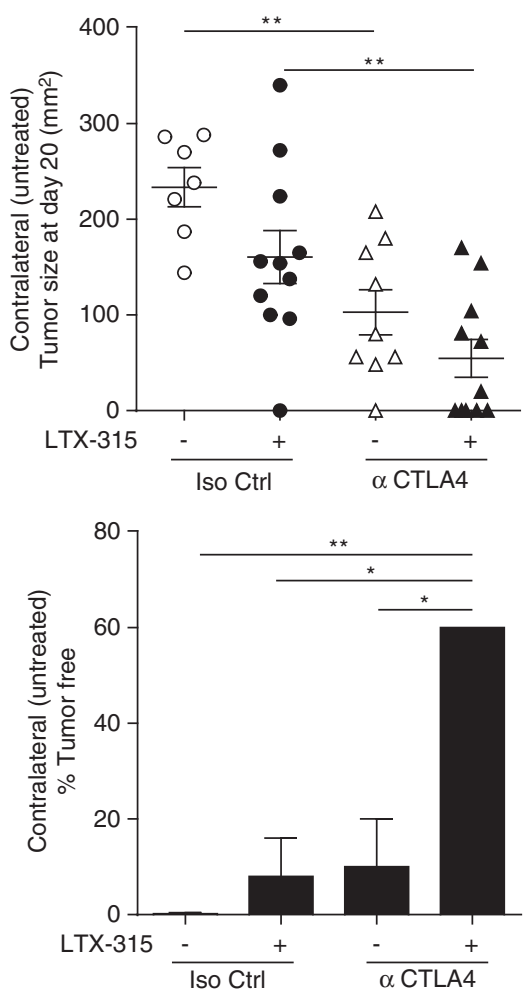
(9D9) or $250 \mu \mathrm{g}$ of anti-PD-1 mAb (RMP1-14), or relevant isotype control (MPC11 or 2A3). Mice were injected three times at 3-day intervals with anti-CTLA4 or antiPD-1 i.p. or two times peritumorally for anti-CTLA4 mAb $(50 \mu \mathrm{g})$, and tumor size was routinely monitored by means of a caliper. In order to evaluate the synergistic effect of LTX-315 and anti-CTLA4, several experimental settings were performed, as detailed in figure legends. Of note, for local peritumoral injections of anti-CTLA4
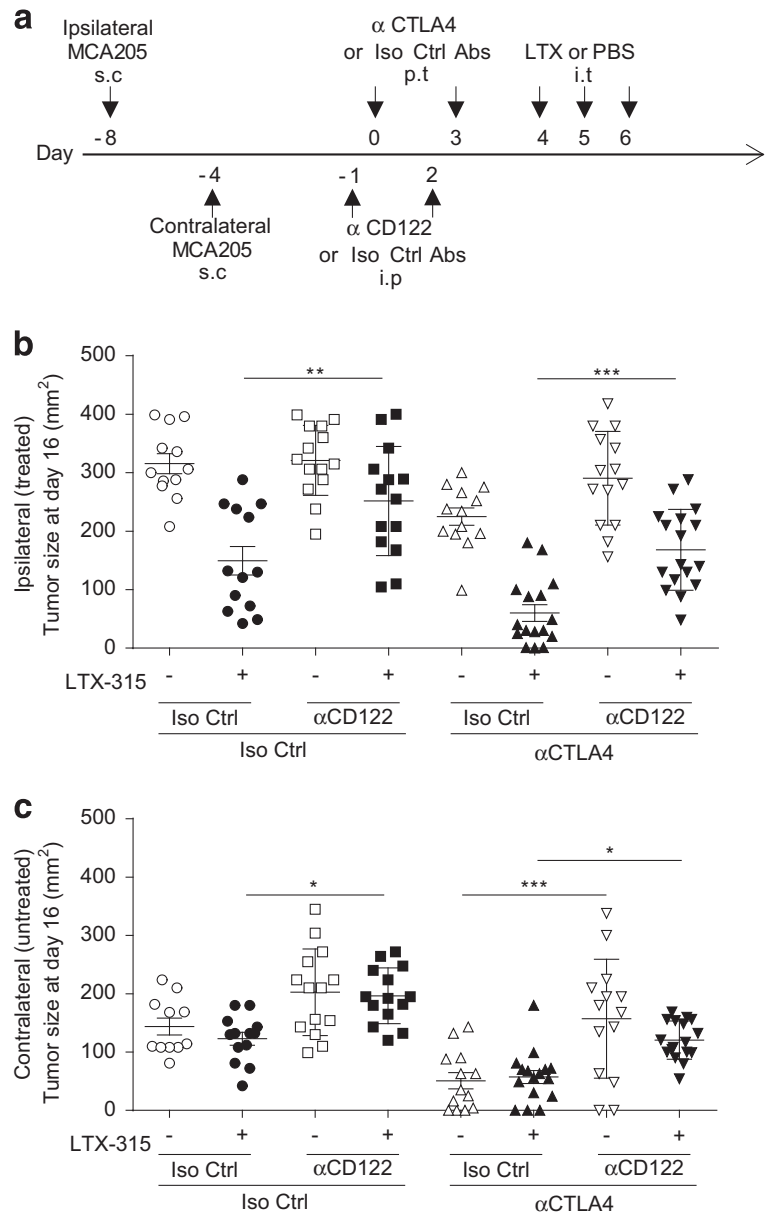

Figure 8 Role of CD122 in the local and distant effects of LTX-315. (a) Experimental setting combining LTX-315 \pm anti-CTLA4 mAb in the presence of a neutralization of CD122 receptors, with bilateral tumor challenge. Scheduling and routing of the combinatorial regimen as detailed in Materials and Methods section. $s$. c., subcutaneous; i.p., intraperitoneal; p.t., peritumoral; i.t., intratumoral. (b and c) Tumor sizes at killing in the ipsilateral (treated, b) or contralateral (untreated, c) sarcoma in the context of local delivery of the immune checkpoint-targeting antibody (anti-CTLA4 mAb: $50 \mu \mathrm{g} /$ mouse, two injections) or isotype control mAb injected before LTX-315 during a systemic neutralization of CD122 with specific mAb or isotype control mAb performed the day before CTLA4 blockade. In all graphs, two experiments comprising 7-9 mice per group are shown. One-way ANOVA followed by Tukey's test: ${ }^{*} P<0.05,{ }^{* \star} P<0.01,{ }^{* \star *} P<0.001$; NS, not significant $m A b$, the final dose was $1 / 3$ of that used systemically. In T-cell depletion experiments, anti-CD4 and anti-CD8 mAbs (GK1.5 and 53-6.72, respectively; $200 \mu \mathrm{g}$ per mouse) or their isotype controls (LTF-2 and $2 \mathrm{~A} 3$, respectively) were injected i.p. 3 and 4 days before the first LTX-315 injection and continued every other 7 days. In CD122 neutralizing experiments, anti-CD122 mAb (TM-beta1, $200 \mu \mathrm{g}$ per mouse), or isotype control mAb (LTF-2) was injected i.p. 1 day before each anti-CTLA4 injection. All mAbs for in vivo use were obtained from BioXcell (West Lebanon, NH, USA), using the recommended isotype control mAbs. In experiments examining the abscopal effects of treatment, a second tumor was inoculated into the left, contralateral flank of mice 4 days following inoculation of the first tumor. The reason for this was on two accounts: first, as several products were administered in combination (i.e., LTX-315 and an ICB) against an established tumor, the tumor of the treated site becomes rather advanced, to a stage rendering a contralateral tumor deposit too bulky and resistant to observe any abscopal effect if this was inoculated at the same time as the primary, treated tumor (because of local/peripheral tolerance); second, this attempts to more closely mimic the clinical setting, as when a cancer-bearing patient develops metastases it is hypothesized that he/she is already experienced to tumor antigens of the primary tumor.

Flow cytometry. Tumors and spleens were harvested 7 days after the first injection of LTX-315. Excised tumors were cut into small pieces and digested in RPMI-1640 medium containing Liberase at $25 \mu \mathrm{g} / \mathrm{ml}$ (Roche, Boulogne-Billancourt, France) and DNase 1 at $150 \mathrm{UI} / \mathrm{ml}$ (Roche) for $30 \mathrm{~min}$ at $37^{\circ} \mathrm{C}$. The mixture was subsequently passaged through a $100 \mu \mathrm{m}$ cell strainer. $2 \times 10^{6}$ splenocytes (after red blood cells lysis) or tumor cells were preincubated with purified anti-mouse CD16/CD32 (93; eBioscience, San Diego, CA, USA) for $15 \mathrm{~min}$ at $4^{\circ} \mathrm{C}$, before membrane staining. For intracellular staining, the FoxP3 staining kit (eBioscience) was used. Dead cells were excluded using the Live/Dead Fixable Yellow dead cell stain kit (Life Technologies, Carlsbad, CA, USA). For cytokine staining, cells were stimulated for $4 \mathrm{~h}$ at $37^{\circ} \mathrm{C}$ with $50 \mathrm{ng} / \mathrm{ml}$ of phorbol 12-myristate 13-acetate (PMA; Calbiochem, San Diego, CA, USA), $1 \mu \mathrm{g} / \mathrm{ml}$ of ionomycin (Sigma, St. Louis, MO, USA), and BD Golgi STOP (BD Biosciences, San Jose, CA, USA). Anti-CD45.2 (104), anti-FoxP3 (FJK-16 s), anti-IFN- $\gamma$ (XMG1.2), anti-TNF- $\alpha$ (MP6-XT22), antiCTLA4 (UC10-4B9), anti-PD-1 (J43), and isotype controls rat IgG1 (eBRG1), IgG2a (eBRG2a), IgG2b (eBRG2b), Armenian Hamster lgG (eBio299Arm) were purchased from eBioscience. Anti-CD3 (145-2C11), anti-CD25 (PC61.5.3), anti-Ly-6C (AL-21), KI67 (fluorescein isothiocyanate mouse anti-human KI67 set), rat $\lg \mathrm{G} 1 \kappa$ were obtained from BD Bioscience. Anti-CD4 (GK1.5), anti-CD8 $\beta$ (YTS1567.7), anti-CD11b (M1/70), anti-CD11c (N418), anti-I-A/I-E (M5/114.15.2), rat lgG2a (RTK2758), Armenian Hamster lgG (HTK888), rat lgG2b (RTK4530) were purchased from Biolegend (San Diego, CA, USA). Eight-color flow cytometry analysis was performed with antibodies conjugated to fluorescein isothiocyanate, phycoerythrin, phycoerythrin cyanin 7 , peridinin chlorophyll protein cyanin 5.5 , allophycocyanin cyanin 7, Pacific blue or allophycocyanin. All cells were analyzed on a CyAn ADP (Beckman Coulter, Marseille, France) flow cytometer with FlowJo (Tree Star, Ashland, OR) software.

HMGB1 and Cxcl10 quantification. HMGB1 (IBL International, Hamburg, Germany) and Cxcl10 (R\&D Systems, Minneapolis, MN, USA) were measured by ELISA following the manufacturer's instructions.

Statistical analysis. Data were analyzed with Microsoft Excel (Microsoft Co., Redmont, WA, USA) and Prism 5 (GraphPad, San Diego, CA, USA). Data are presented as means \pm S.E.M. and $P$-values computed by unpaired Student's $t$-tests or one-way ANOVA followed by Tukey's test where applicable. Comparisons of Kaplan-Meier survival curves were performed using the log-rank Mantel-Cox test. All reported tests are two-tailed and were considered significant at $P$-values $<0.05$

Figure 7 LTX-315-based local immunotherapy can be optimized by pre-sensitization of the tumor bed with low dosing of anti-CTLA4 mAb. (a) Experimental setting with bilateral tumor inoculations. Scheduling and routing of mAbs and LTX-315 as detailed in Materials and Methods section. s.c., subcutaneous; p.t., peritumoral; i.t., intratumoral. (b and $\mathbf{c}$ ) Tumor sizes (upper panels) as well as percentages of complete tumor eradication (lower panels) at killing in the ipsilateral (treated, b) or contralateral (untreated, c) sarcoma in the context of local delivery of the immune checkpoint-targeting mAb (anti-CTLA4: $50 \mu \mathrm{g} / \mathrm{mouse}$, two injections) or isotype control mAb injected before LTX-315. (d) Kaplan-Meier curves are depicted in the panel (combined data from two experiments). In all graphs, two experiments comprising 7-9 mice per group are shown. One-way ANOVA followed by Tukey's test: ${ }^{*} P<0.05,{ }^{* \star} P<0.01,{ }^{* \star} P<0.001$; NS, not significant. Comparison of Kaplan-Meier survival curves were performed using the log-rank MantelCox test: ${ }^{\star} P<0.05,{ }^{\star \star *} P<0.001,{ }^{* \star *} P<0.0001$; NS, not significant 


\section{Conflict of Interest}

The authors declare no conflict of interest.

Acknowledgements. LZ and GK were supported by the Ligue contre le Cancer (équipes labelisées); Agence National de la Recherche (ANR) - Projets blancs; ANR under the frame of E-Rare-2, the ERA-Net for Research on Rare Diseases; Association pour la recherche sur le cancer (ARC); Cancéropôle lle-de-France; Institut National du Cancer (INCa); Fondation Bettencourt-Schueller; Fondation de France; Fondation pour la Recherche Médicale (FRM); the European Commission (ArtForce); the European Research Council (ERC); the LabEx Immuno-Oncology; the SIRIC Stratified Oncology Cell DNA Repair and Tumor Immune Elimination (SOCRATE); the SIRIC Cancer Research and Personalized Medicine (CARPEM); the Swiss Bridge Foundation, ISREC and the Paris Alliance of Cancer Research Institutes (PACRI). This project was supported by Lytix Biopharma Ltd. We thank $P$ Gonin in Service Commun d'Expérimentation Animale for providing excellent animal colony care.

1. Marabelle $A$, Kohrt $H$, Caux $C$, Levy R. Intratumoral immunization: a new paradigm for cancer therapy. Clin Cancer Res 2014; 20: 1747-1756.

2. Church SE, Galon J. Tumor microenvironment and immunotherapy: the whole picture is better than a glimpse. Immunity 2015; 43: 631-633.

3. Fridman WH, Pages F, Sautes-Fridman C, Galon J. The immune contexture in human tumours: impact on clinical outcome. Nat Rev Cancer 2012; 12: 298-306.

4. Marabelle A, Kohrt H, Sagiv-Barfi I, Ajami B, Axtell RC, Zhou G et al. Depleting tumorspecific Tregs at a single site eradicates disseminated tumors. J Clin Invest 2013; 123: 2447-2463.

5. Brody JD, Ai WZ, Czerwinski DK, Torchia JA, Levy M, Advani RH et al. In situ vaccination with a TLR9 agonist induces systemic lymphoma regression: a phase I/II study. J Clin Oncol 2010; 28: 4324-4332.

6. Kim Y, Spitz GS, Veturi U, Lach FP, Auerbach AD, Smogorzewska A. Regulation of multiple DNA repair pathways by the Fanconi anemia protein SLX4. Blood 2013; 121: 54-63.

7. Postow MA, Callahan MK, Barker CA, Yamada Y, Yuan J, Kitano S et al. Immunologic correlates of the abscopal effect in a patient with melanoma. N Engl J Med 2012; 366: 925-931.

8. Selby MJ, Engelhardt JJ, Quigley M, Henning KA, Chen T, Srinivasan M et al. Anti-CTLA-4 antibodies of IgG2a isotype enhance antitumor activity through reduction of intratumoral regulatory T cells. Cancer Immunol Res 2013; 1: 32-42.

9. Harrington KJ, Puzanov I, Hecht JR, Hodi FS, Szabo Z, Murugappan S et al. Clinical development of talimogene laherparepvec (T-VEC): a modified herpes simplex virus type-1derived oncolytic immunotherapy. Exp Rev Anticancer Ther 2015; 15: 1389-1403.

10. Zasloff M. Antimicrobial peptides of multicellular organisms. Nature 2002; 415: 389-395.

11. Rosenfeld Y, Shai Y. Lipopolysaccharide (endotoxin)-host defense antibacterial peptides interactions: role in bacterial resistance and prevention of sepsis. Biochim Biophys Acta 2006; 1758: 1513-1522.

12. Brown KL, Hancock RE. Cationic host defense (antimicrobial) peptides. Curr Opin Immunol 2006; 18: 24-30.

13. Wieczorek M, Jenssen H, Kindrachuk J, Scott WR, Elliott M, Hilpert K et al. Structural studies of a peptide with immune modulating and direct antimicrobial activity. Chem Biol 2010; 17: 970-980.

14. Schweizer F. Cationic amphiphilic peptides with cancer-selective toxicity. Eur J Pharmacol 2009; 625: 190-194.

15. Hoskin DW, Ramamoorthy A. Studies on anticancer activities of antimicrobial peptides Biochim Biophys Acta 2008; 1778: 357-375.

16. Mader JS, Hoskin DW. Cationic antimicrobial peptides as novel cytotoxic agents for cancer treatment. Expert Opin Investig Drugs 2006; 15: 933-946.

17. Papo N, Shai Y. Host defense peptides as new weapons in cancer treatment. Cell Mol Life Sci 2005; 62: 784-790.

18. Masson PL, Heremans JF. Lactoferrin in milk from different species. Comp Biochem Physiol B 1971; 39: 119-129.

19. Yoo YC, Watanabe R, Koike Y, Mitobe M, Shimazaki K, Watanabe S et al. Apoptosis in human leukemic cells induced by lactoferricin, a bovine milk protein-derived peptide: involvement of reactive oxygen species. Biochem Biophys Res Commun 1997; 237: 624-628.

20. Yoo YC, Watanabe S, Watanabe R, Hata K, Shimazaki K, Azuma I. Bovine lactoferrin and lactoferricin, a peptide derived from bovine lactoferrin, inhibit tumor metastasis in mice. Jpn J Cancer Res 1997; 88: 184-190.
21. Eliassen LT, Berge G, Sveinbjornsson B, Svendsen JS, Vorland LH, Rekdal O. Evidence for a direct antitumor mechanism of action of bovine lactoferricin. Anticancer Res 2002; 22 2703-2710.

22. Eliassen LT, Berge G, Leknessund A, Wikman M, Lindin I, Lokke $C$ et al. The antimicrobia peptide, lactoferricin $B$, is cytotoxic to neuroblastoma cells in vitro and inhibits xenograft growth in vivo. Int J Cancer 2006; 119: 493-500.

23. Mader JS, Salsman J, Conrad DM, Hoskin DW. Bovine lactoferricin selectively induces apoptosis in human leukemia and carcinoma cell lines. Mol Cancer Ther 2005; 4: 612-624.

24. Mader JS, Richardson A, Salsman J, Top D, de Antueno R, Duncan R et al. Bovine lactoferricin causes apoptosis in Jurkat T-leukemia cells by sequential permeabilization of the cell membrane and targeting of mitochondria. Exp Cell Res 2007; 313: 2634-2650.

25. Haug BE, Camilio KA, Eliassen LT, Stensen W, Svendsen JS, Berg K et al. Discovery of a 9-mer cationic peptide (LTX-315) as a potential first in class oncolytic peptide. J Med Chem (e-pub ahead of print 16 March 2016).

26. Zhou H, Forveille S, Sauvat A, Sica V, Izzo V, Durand S et al. The oncolytic peptide LTX-315 kills cancer cells through Bax/Bak-regulated mitochondrial membrane permeabilization. Oncotarget 2015; 6: 26599-26614.

27. Eike LM, Yang N, Rekdal O, Sveinbjornsson B. The oncolytic peptide LTX-315 induces cell death and DAMP release by mitochondria distortion in human melanoma cells. Oncotarget 2015; 6: 34910-34923.

28. Camilio KA, Berge G, Ravuri CS, Rekdal O, Sveinbjornsson B. Complete regression and systemic protective immune responses obtained in B16 melanomas after treatment with LTX-315. Cancer Immunol Immunother 2014; 63: 601-613.

29. Camilio KA, Rekdal O, Sveinbjornsson B. LTX-315 (oncopore): a short synthetic anticancer peptide and novel immunotherapeutic agent. Oncoimmunology 2014; 3: e29181.

30. Forveille S, Zhou H, Sauvat A, Bezu L, Muller K, Liu P et al. The oncolytic peptide LTX-315 triggers necrotic cell death. Cell Cycle 2015; 14: 3506-3512.

31. Spicer JAA, Brunsvig P, Saunders A, Olsen WM, Nicolaisen B, Rekdal O et al. Intratumoural treatment with LTX-315, an oncolytic peptide immunotherapy, in patients with advanced metastatic disease induces CD8 effector cells and regression in some injected tumours. Eur Cancer Congr 20152015

32. Apetoh L, Ghiringhelli F, Tesniere A, Obeid M, Ortiz C, Criollo A et al. Toll-like receptor 4-dependent contribution of the immune system to anticancer chemotherapy and radiotherapy. Nat Med 2007; 13: 1050-1059.

33. Sistigu A, Yamazaki T, Vacchelli E, Chaba K, Enot DP, Adam J et al. Cancer cell-autonomous contribution of type I interferon signaling to the efficacy of chemotherapy. Nat Med 2014; 20: 1301-1309.

34. Hannani D, Vetizou M, Enot D, Rusakiewicz S, Chaput N, Klatzmann D et al. Anticancer immunotherapy by CTLA-4 blockade: obligatory contribution of IL-2 receptors and negative prognostic impact of soluble CD25. Cell Res 2015; 25: 208-224.

35. Zhou H, Forveille S, Sauvat A, Yamazaki T, Senovilla L, Ma Y et al. The oncolytic peptide LTX-315 triggers immunogenic cell death. Cell Death Differ 2015; 7: e2134.

36. Ngiow SF, Young A, Jacquelot N, Yamazaki T, Enot D, Zitvogel $L$ et al. A threshold level of intratumor CD8+ T-cell PD1 expression dictates therapeutic response to Aanti-PD1. Cancer Res 2015; 75: 3800-3811.

37. Vacchelli E, Ma Y, Baracco EE, Sistigu A, Enot DP, Pietrocola F et al. Chemotherapyinduced antitumor immunity requires formyl peptide receptor 1 . Science 2015; 350: 972-978.

38. Roux S, Apetoh L, Chalmin F, Ladoire S, Mignot G, Puig PE et al. CD4+CD25+ Tregs contro the TRAIL-dependent cytotoxicity of tumor-infiltrating DCs in rodent models of colon cancer. $J$ Clin Invest 2008; 118: 3751-3761.

39. Klug F, Prakash H, Huber PE, Seibel T, Bender N, Halama N et al. Low-dose irradiation programs macrophage differentiation to an $\mathrm{NOS}(+) / \mathrm{M} 1$ phenotype that orchestrates effective T cell immunotherapy. Cancer Cell 2013; 24: 589-602.

40. Viaud S, Saccheri F, Mignot G, Yamazaki T, Daillere R, Hannani D et al. The intestinal microbiota modulates the anticancer immune effects of cyclophosphamide. Science 2013; 342: $971-976$.

41. lida N, Dzutsev A, Stewart CA, Smith L, Bouladoux N, Weingarten RA et al. Commensal bacteria control cancer response to therapy by modulating the tumor microenvironment. Science 2013; 342: 967-970

42. Sivan A, Corrales L, Hubert N, Williams JB, Aquino-Michaels K, Earley ZM et al. Commensal bifidobacterium promotes antitumor immunity and facilitates anti-PD-L1 efficacy. Science 2015; 350: 1084-1089.

43. Vetizou M, Pitt JM, Daillere R, Lepage P, Waldschmitt N, Flament C et al. Anticancer immunotherapy by CTLA-4 blockade relies on the gut microbiota. Science 2015; 350: 1079-1084.

44. Urbaniak C, Cummins J, Brackstone M, Macklaim JM, Gloor GB, Baban CK et al. Microbiota of human breast tissue. Appl Environ Microbiol 2014; 80: 3007-3014. 